\title{
Module sectional category of products
}

\author{
J. G. Carrasquel ${ }^{1}$. P.-E. Parent ${ }^{2}$ • \\ L. Vandembroucq ${ }^{3}$
}

Received: 22 January 2016 / Accepted: 27 September 2017 / Published online: 12 October 2017

(C) Tbilisi Centre for Mathematical Sciences 2017

\begin{abstract}
Adapting a result of Félix-Halperin-Lemaire concerning the LusternikSchnirelmann category of products, we prove the additivity of a rational approximation for Schwarz's sectional category with respect to products of certain fibrations.
\end{abstract}

Keywords Rational homotopy $\cdot$ Sectional category $\cdot$ Topological complexity

Mathematics Subject Classification 55M30 - 55P62

\section{Introduction}

The sectional category [12] (or Schwarz genus) of a fibration $p: E \rightarrow X$, secat $(p)$, is the smallest integer $m$ such that $X$ admits a cover by $(m+1)$ open sets on each of which a local section for $p$ exists. This homotopy invariant is a generalization of

Communicated by Pascal Lambrechts.

$凶 \quad$ J. G. Carrasquel

jgcarras@amu.edu.pl; jgcarras@gmail.com

P.-E. Parent

pparent@uottawa.ca

L. Vandembroucq

lucile@math.uminho.pt

1 Faculty of Mathematics and Computer Science, Adam Mickiewicz University, Umultowska 87, 61-614 Poznań, Poland

2 Department of Mathematics and Statistics, University of Ottawa, 585 King Edward Ave., Ottawa, ON K1N 6N5, Canada

3 Centro de Matemática, Universidade do Minho, Campus de Gualtar, 4710-057 Braga, Portugal 
the well-known Lusternik-Schnirelmann (L.-S.) category [10] of a path-connected space $X$, cat $(X)$, as the latter is the sectional category of the path fibration $P X \rightarrow X$, $\alpha \mapsto \alpha(1)$, where $P X$ is the space of paths starting at the base point.

One of the most important results of [5] says that, if $X$ and $Y$ are simply connected rational spaces of finite type, then $\operatorname{cat}(X \times Y)=\operatorname{cat}(X)+\operatorname{cat}(Y)$. This was achieved by first proving the analogous result for the lower bound module L.-S. category, mcat $(X)$, of cat $(X)$ using differential graded (DG) module techniques. It was then lifted to rational category using Hess' theorem [9]. We propose to apply similar DG-module techniques to the lower bound msecat $(p)$ of secat $(p)$ called module sectional category and introduced in [7].

Throughout this paper we consider fibrations whose base and total space have the homotopy type of simply connected $\mathrm{CW}$-complexes of finite type. Our main result is

Theorem 1 Let $p$ and $p^{\prime}$ be two fibrations. If either $p$ or $p^{\prime}$ admits a homotopy retraction, then

$$
\operatorname{msecat}\left(p \times p^{\prime}\right)=\operatorname{msecat}(p)+\operatorname{msecat}\left(p^{\prime}\right) .
$$

Recall the important particular case of sectional category provided by Farber's (higher) topological complexity $[4,11]$ of a space $X, \mathrm{TC}_{n}(X)=\operatorname{secat}\left(\pi_{n}\right)$, where the considered fibration $\pi_{n}: X^{[1, n]} \rightarrow X^{n}$ is given by $\pi_{n}(\alpha)=(\alpha(1), \alpha(2), \ldots, \alpha(n))$. Consequently, the module invariant associated to (higher) topological complexity, i.e.,

$$
\operatorname{mTC}_{n}(X):=\operatorname{msecat}\left(\pi_{n}\right)
$$

is additive on products. Namely

Corollary 2 Let $X$ and $Y$ be two spaces. Then

$$
\operatorname{mTC}_{n}(X \times Y)=\operatorname{mTC}_{n}(X)+\operatorname{mTC}_{n}(Y) .
$$

These results are improvements over [2] as only one of the two fibrations of Theorem 1 needs a homotopy retraction and the Poincaré duality assumption is no longer required.

\section{Preliminaries}

This section contains a brief summary of the DG-modules techniques that will be used (see [6] for further details). Let $(A, d)$ be a commutative differential graded algebra over $\mathbb{Q}(\mathrm{cdga})$. An $(A, d)$-module is a chain complex $(M, d)$ together with a degree 0 action of $A$ satisfying $d(a x)=(d a) x+(-1)^{|a|} a(d x)$. A semifree extension of an $(A, d)$-module $(M, d)$ is an $(A, d)$-module of the form $(M \oplus A \otimes U, d)$ where the action is the one of the direct sum, the differential on $M$ is the differential of $(M, d)$, and $U$ admits a direct sum decomposition $U=\oplus_{i=0}^{\infty} U_{i}$ such that $d\left(U_{0}\right) \subset M$ and $d\left(U_{n}\right) \subset M \oplus A \otimes\left(\oplus_{i=0}^{n-1} U_{i}\right)$ for $n \geq 1$. A semifree $(A, d)$-module is a semifree extension $(A \otimes U, d)$ of the trivial $(A, d)$-module 0 and the data of a quasi-isomorphism 
$(A \otimes U, d) \stackrel{\simeq}{\rightarrow}(M, d)$ is called a semifree resolution of $(M, d)$. The category of $(A, d)$-modules is a proper closed model category in which semifree extensions are cofibrations (see, for instance, [7, Theorem 4.1]). Two $(A, d)$-module morphisms $\phi, \psi:(M, d) \rightarrow(N, d)$ are homotopic if there is an $A$-linear map $\theta: M \rightarrow N$ of degree -1 such that $\phi-\psi=d \theta+\theta d$. We will frequently use the fact that any $(A, d)$-module morphism $\varphi:(M, d) \rightarrow(N, d)$ can be decomposed as (the inclusion of) a semifree extension followed by a quasi-isomorphism as well as the following lifting lemma. Given a solid arrow commutative diagram of $(A, d)$-modules of the form

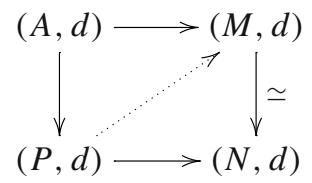

in which the morphism $(A, d) \rightarrow(P, d)$ is a semifree extension, there is an $(A, d)$ module morphism $(P, d) \rightarrow(M, d)$ making commutative the upper triangle and homotopy commutative (rel. $A)$ the lower triangle. A morphism of $(A, d)$-modules $\varphi:(M, d) \rightarrow(N, d)$ is said to have a homotopy retraction if there exists a commutative diagram of $(A, d)$-modules,

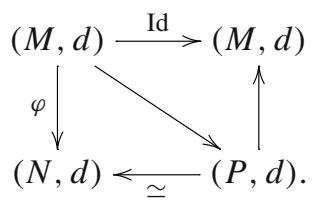

If $M$ is an $(A, d)$-module, the module $M^{\#}=\operatorname{hom}(M, \mathbb{Q})$ admits an $(A, d)$-module structure with action $(a \varphi)(x)=(-1)^{|a| \cdot|\varphi|} \varphi(a x)$ and differential $d \varphi=(-1)^{|\varphi|} \varphi \circ d$. If $N$ is an $(A, d)$-module, then the module $M \otimes_{A} N$ admits an $(A, d)$-module structure with action $a(m \otimes n)=(a m) \otimes n$ and differential $d(m \otimes n)=d m \otimes n+(-1)^{|m|} m \otimes d n$. If $P$ is $(A, d)$-semifree and if $\eta$ is a quasi-isomorphism of $(A, d)$-modules then $\eta \otimes_{A} \operatorname{Id}_{P}$ and $\operatorname{Id}_{P} \otimes_{A} \eta$ are also quasi-isomorphisms.

The following lemma is an adaptation of a central idea of [5].

Lemma 3 Let $\varphi:(A, d) \rightarrow(B, d)$ be a surjective cdga morphism with kernel $K$ and A of finite type. The morphism $\varphi$ admits a homotopy retraction of $(A, d)$-modules if and only if for any $(A, d)$-semifree resolution $\eta: P \stackrel{\simeq}{\longrightarrow} A^{\#}$, the projection

$$
\varrho: P \longrightarrow \frac{P}{K \cdot P}
$$

is injective in homology. 
Proof Suppose that $\varphi$ admits a homotopy retraction of $(A, d)$-modules. This means that there exists a homotopy commutative diagram of $(A, d)$-modules of the form

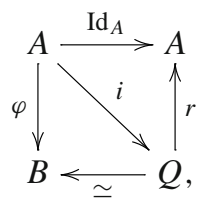

where $Q$ is an $(A, d)$-semifree resolution of $B$. Now let $\eta: P \stackrel{\simeq}{\longrightarrow} A^{\#}$ be an $(A, d)$ semifree resolution. By applying $-\otimes_{A} P$ to the diagram above, we get

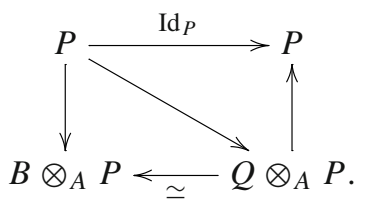

Since $B$ and $\frac{A}{K}$ are isomorphic cdgas, we have $B \otimes_{A} P=\frac{P}{K \cdot P}$. Hence the left hand morphism is simply the projection $\varrho: P \rightarrow \frac{P}{K \cdot P}$. The diagram shows that $\varrho$ admits a homotopy retraction of $(A, d)$-modules. Hence it is injective in homology.

Conversely, suppose that $\varrho$ is injective in homology. Since $A$ is of finite type, $\eta^{\#}: A \rightarrow P^{\#}$ is also an $(A, d)$-semifree resolution. Moreover,

$$
\varrho^{\#}:\left(\frac{P}{K \cdot P}\right)^{\#} \rightarrow P^{\#}
$$

is surjective in homology. Hence there exists a cycle $\gamma \in\left(\frac{P}{K \cdot P}\right)^{\#}$ such that $[\gamma \circ$ $\varrho]=\left[\eta^{\#}(1)\right]$. Now define an $(A, d)$-module morphism $\alpha: A \rightarrow\left(\frac{P}{K \cdot P}\right)^{\#}$ by setting $\alpha(1)=\gamma$. Then $\varrho^{\#} \circ \alpha$ is a quasi-isomorphism. To finish the proof, we observe that $K \cdot\left(\frac{P}{K \cdot P}\right)^{\#}=0$. Hence the map $\varrho^{\#} \circ \alpha$ factors through $\varphi$ as $B=A / K$. Let $A \stackrel{i}{\rightarrow} Q \stackrel{\simeq}{\rightarrow} B$ be a decomposition of $\varphi$ as a semifree extension followed by a quasiisomorphism. Applying the lifting lemma to the solid arrow commutative diagram

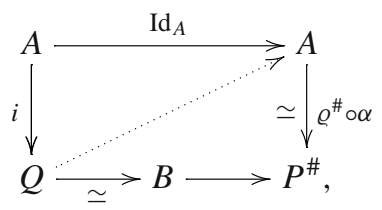

we obtain the desired homotopy $(A, d)$-module retraction for $\varphi$. 


\section{The invariant msecat $(p)$}

Let us denote by $p_{m}: J_{X}^{m}(E) \rightarrow X$ the join of $m+1$ copies of a fibration $p: E \rightarrow X$. As is well-known [12], $\sec a t(p) \leq m$ if and only if $p_{m}$ admits a homotopy section. By definition, msecat $(p)$ is the smallest $m$ such that $A_{P L}\left(p_{m}\right)$ admits a homotopy retraction of $A_{P L}(X)$-modules, where $A_{P L}$ denotes Sullivan's functor of piecewise linear forms [13].

Let $\varphi:(A, d) \rightarrow(B, d)$ be any cdga model of $p$ and

$$
(A, d) \hookrightarrow(A \otimes(\mathbb{Q} \oplus U), d) \stackrel{\xi}{\rightarrow}(B, d) .
$$

a factorization in the category of $(A, d)$-modules of $\varphi$ as the inclusion of a semifree extension followed by a quasi-isomorphism $\xi$. We refer to the inclusion as a semifree model of $p$. For $x \in U$, we write $d x=d_{0} x+d_{+} x$, where $d_{0} x \in A$ and $d_{+} x \in A \otimes U$. We notice that, if $\varphi$ is surjective, then the quasi-isomorphism $\xi$ can be constructed to satisfy $\xi(U)=0$, which implies that $d_{0} x \in \operatorname{ker} \varphi$ for $x \in U$. Recall that the $n^{\text {th }}$-suspension $s^{-n} V$ of a graded vector space $V$ is defined by $\left(s^{-n} V\right)^{i}=V^{i-n}$.

According to [7] (Thm 5.4, p.135), $\operatorname{msecat}(p)$ is the least $m$ such that the following $(A, d)$-semifree model of $p_{m}$

$$
j_{m}:(A, d) \rightarrow \underbrace{\left(A \otimes\left(\mathbb{Q} \oplus s^{-m} U^{\otimes m+1}\right), D\right)}_{J_{m}} .
$$

admits a retraction of $(A, d)$-modules, where the differential $D$ is given by

$$
\begin{aligned}
& D\left(s^{-m} x_{0} \otimes \cdots \otimes x_{m}\right)=(-1)^{\sum_{k=1}^{m}\left(k\left|x_{m-k}\right|+k-1\right)} d_{0} x_{0} \cdots d_{0} x_{m} \\
& +\sum_{i=0}^{m} \sum_{j_{i}}(-1)^{\left(\left|a_{i j}\right|+1\right)\left(\left|x_{0}\right|+\cdots+\left|x_{i-1}\right|+m\right)} a_{i j_{i}} \otimes s^{-m} x_{0} \otimes \cdots \otimes x_{i j_{i}} \otimes \cdots \otimes x_{m}
\end{aligned}
$$

for $x_{0}, \ldots, x_{m} \in U$ and $d_{+} x_{i}=\sum_{j_{i}} a_{i j_{i}} \otimes x_{i j_{i}}$ with $a_{i j_{i}} \in A$ and $x_{i j_{i}} \in U$.

Using the following notation (suggested by the standard rules of signs)

$$
s^{-m} x_{0} \otimes \cdots \otimes d_{+} x_{i} \otimes \cdots \otimes x_{m}:=\sum_{j_{i}} \sigma_{i j_{i}} a_{i j_{i}} \otimes s^{-m} x_{0} \otimes \cdots \otimes x_{i j_{i}} \otimes \cdots \otimes x_{m},
$$

we can write $D_{+}\left(s^{-m} x_{0} \otimes \cdots \otimes x_{m}\right)$ as

$$
D_{+}\left(s^{-m} x_{0} \otimes \cdots \otimes x_{m}\right)=(-1)^{m} \sum_{i=0}^{m} \sum_{j_{i}} \tau_{i} s^{-m} x_{0} \otimes \cdots \otimes d_{+} x_{i} \otimes \cdots \otimes x_{m},
$$

where $\sigma_{i j_{i}}:=(-1)^{\left|a_{i j_{i}}\right|\left(\left|x_{0}\right|+\cdots+\left|x_{i-1}\right|+m\right)}$ and $\tau_{i}:=(-1)^{\left(\left|x_{0}\right|+\cdots+\left|x_{i-1}\right|\right)}$.

When the fibration $p: E \rightarrow X$ is endowed with a homotopy retraction, there exists a surjective cdga model of $p$ which is a retraction of a cdga cofibration (see, 
for instance, [3, Section 5.1] for an explicit construction). Such a model is called an $s$-model. We will use the following result from [1].

Theorem 4 ([1, Theorem 3.3]) Let p be a fibration endowed with a homotopy retraction. For any $s$-model $\varphi: A \rightarrow \frac{A}{K}$ of $p$, $\operatorname{msecat}(p)$ is the smallest $m$ for which the projection $\rho_{m}: A \rightarrow \frac{A}{K^{m+1}}$ admits a homotopy retraction of $(A, d)$-modules.

By using this result together with Lemma 3, we obtain the following new characterization of $\operatorname{msecat}(p)$ when $p$ admits a homotopy retraction.

Proposition 5 Let $p: E \rightarrow X$ be a fibration endowed with a homotopy retraction, $\varphi: A \rightarrow \frac{A}{K}$ an s-model for $p$ and $(A, d) \rightarrow(A \otimes(\mathbb{Q} \oplus U), d)$ a semifree extension for $\varphi$, as in (1). Let also $\eta: P \stackrel{\simeq}{\longrightarrow} A^{\#}$ be an $(A, d)$ semifree resolution. Then the following are equivalent

(i) $\operatorname{msecat}(p) \leq m$,

(ii) the morphism $\operatorname{Id}_{P} \otimes_{A} j_{m}: P \rightarrow P \otimes\left(\mathbb{Q} \oplus s^{-m} U^{\otimes m+1}\right)$ is injective in homology,

(iii) the projection $P \rightarrow \frac{P}{K^{m+1} \cdot P}$ is injective in homology.

Proof It is clear that $(i)$ implies $(i i)$. From the proof of [1, Theorem 3.3], there is a diagram

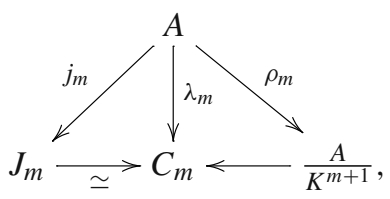

where the map $\lambda_{m}: A \rightarrow C_{m}$ is a model of $p_{m}: J_{X}^{m}(E) \rightarrow X$, the left hand triangle is commutative up to a homotopy of $(A, d)$-modules, and the right hand triangle is strictly commutative. Applying $\operatorname{Id}_{P} \otimes_{A}-$ to the previous diagram, we get the following diagram of $(A, d)$-modules:

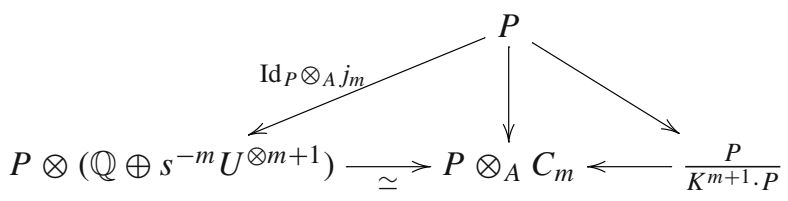

where the left hand triangle is commutative up to a homotopy of $(A, d)$-modules and the right hand triangle is strictly commutative, which yields $(i i) \Rightarrow(i i i)$. Finally the implication $(\mathrm{iii}) \Rightarrow(\mathrm{ii})$ follows from Lemma 3 applied to $\rho_{m}$.

\section{The main result}

Finally, we present a proof of the additivity of module sectional category when only one of the fibrations admits homotopy retraction.

We first notice that one of the inequalities of Theorem 1 follows in general: 
Proposition 6 Let $p: E \rightarrow X$ and $p^{\prime}: E^{\prime} \rightarrow X^{\prime}$ be two fibrations. We have

$$
\operatorname{msecat}\left(p \times p^{\prime}\right) \leq \operatorname{msecat}(p)+\operatorname{msecat}\left(p^{\prime}\right) .
$$

Proof In [8, Section 7.2], maps $\psi_{n, m}^{E, E^{\prime}}$ producing a commutative diagram of the following form are constructed:

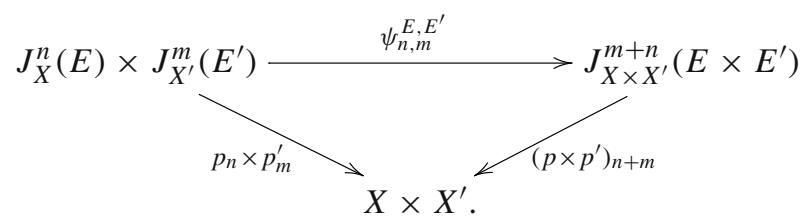

By applying $A_{P L}$ to this diagram, we can establish that, if $\operatorname{msecat}(p) \leq m$ and $\operatorname{msecat}\left(p^{\prime}\right) \leq n$ then msecat $\left(p \times p^{\prime}\right) \leq m+n$.

In order to prove our main result (Theorem 1), it remains to establish the inequality $\operatorname{msecat}\left(p \times p^{\prime}\right) \geq \operatorname{msecat}(p)+\operatorname{msecat}\left(p^{\prime}\right)$ under the additional assumption that one of the fibration, say $p$, admits a homotopy retraction. We notice that, if both fibrations would admit a homotopy retraction, a direct adaptation of the strategy of [5] together with Proposition 5 would give a proof of this inequality. The following less immediate adaptation of [5] provides a proof when only $p$ admits a homotopy retraction.

Proof (Proof of Theorem 1) Take an s-model $\varphi$ for $p$ and an $(A, d)$-semifree extension $(A \otimes(\mathbb{Q} \oplus U), d)$ of $\varphi$ such that $d_{0}(x) \in K=\operatorname{ker} \varphi$ for $x \in U$. Let also $(B, d) \rightarrow$ $(B \otimes(\mathbb{Q} \oplus V), d)$ be a $(B, d)$-semifree model of $p^{\prime}$. Then $p \times p^{\prime}$ is modeled by the tensor product of the two semifree extensions which gives a semifree extension of $(A \otimes B, d)$-modules that we write as follows

$$
A \otimes B \rightarrow A \otimes B \otimes(\mathbb{Q} \oplus Z), \quad \text { where } \quad Z=U \oplus V \oplus U \otimes V .
$$

In order to prove the statement, we suppose $\operatorname{msecat}(p)=m$ and $\operatorname{msecat}\left(p \times p^{\prime}\right)=$ $m+n$ and show that msecat $\left(p^{\prime}\right) \leq n$.

Let $P \stackrel{\simeq}{\longrightarrow} A^{\#}$ be an $(A, d)$-semifree resolution. Since msecat $(p)=m$ we know from Proposition 5 that there exists $\Omega \in H\left(K^{m} \cdot P\right)$ which is not trivial in $H(P)$. Then there exist a cocyle $\omega \in K^{m} \cdot P$ representing $\Omega$ in $H(P)$ and $\theta \in P \otimes s^{-(m-1)} U^{\otimes m}$ such that $d \theta=\omega$. As a chain complex, we can write $P=\omega \cdot \mathbb{Q} \oplus S$ where $d(S) \subset S$, and we define the following linear map of degree $-|\omega|$ :

$$
I_{\omega}: P \rightarrow \mathbb{Q}, \quad I_{\omega}(\omega)=1, \quad I_{\omega}(S)=0
$$

This map commutes with differentials. Now write the element $\theta \in P \otimes s^{-(m-1)} U^{\otimes m}$ as

$$
\theta=\sum_{i} q_{i} \otimes s^{-(m-1)} x_{i}
$$


with $q_{i} \in P$ and $x_{i} \in U^{\otimes m}$. Since $d \theta=\omega$ we have $d_{+} \theta=0$ and $d_{0} \theta=\omega$.

Let $\psi: B \otimes\left(\mathbb{Q} \oplus s^{-n} V^{\otimes n+1}\right) \rightarrow P \otimes B \otimes\left(\mathbb{Q} \oplus s^{-m-n} Z^{\otimes m+n+1}\right)$ be the $B$-linear map of degree $|\omega|$ given by $\psi(1)=\omega \otimes 1$ and, for $y \in V^{\otimes n+1}$,

$$
\psi\left(s^{-n} y\right)=-(-1)^{n|\omega|} \sum_{i}(-1)^{(n+1)\left|q_{i}\right|} q_{i} \otimes 1 \otimes s^{-m-n} x_{i} \otimes y
$$

and extended to $B \otimes\left(\mathbb{Q} \oplus s^{-n} V^{\otimes n+1}\right)$ by the rule $\psi(b \cdot x)=(-1)^{|b||\omega|} b \cdot \psi(x)$. Notice that the structure of $(B, d)$-module on $P \otimes B \otimes\left(\mathbb{Q} \oplus s^{-m-n} Z^{\otimes m+n+1}\right)$ is given by $b \cdot\left(q \otimes b^{\prime} \otimes z\right)=(-1)^{|q||b|} q \otimes b b^{\prime} \otimes z$. In particular $\psi(b)=\omega \otimes b$. Let us now see that $\psi$ commutes with differentials, that is $\psi \circ d=(-1)^{|\omega|} d \circ \psi$. Since $\psi$ is $B$-linear and since $\omega$ is a cocycle we only have to see that

$$
d \psi\left(s^{-n} y\right)=(-1)^{|\omega|} \psi\left(d s^{-n} y\right)
$$

for each $y \in V^{\otimes n+1}$. Writing the differential of $P \otimes B \otimes\left(\mathbb{Q} \oplus s^{-m-n} Z^{\otimes m+n+1}\right)$ as

$$
d=d_{0}+d_{+} \in P \otimes B \oplus P \otimes B \otimes s^{-m-n} Z^{\otimes m+n+1}
$$

we can check that

$-d_{0} \psi\left(s^{-n} y\right)=(-1)^{|\omega|} \psi\left(d_{0} s^{-n} y\right)$ using the fact that $d_{0} \theta=\omega$, and

$-d_{+} \psi\left(s^{-n} y\right)=(-1)^{|\omega|} \psi\left(d_{+} s^{-n} y\right)$ using the fact that $d_{+} \theta=0$.

From the assumption msecat $\left(p \times p^{\prime}\right)=m+n$ we know that the morphism

$$
j_{m+n}^{A \otimes B}: A \otimes B \rightarrow A \otimes B \otimes\left(\mathbb{Q} \oplus s^{-m-n} Z^{\otimes m+n+1}\right) .
$$

admits a retraction $r$ of $(A \otimes B, d)$-modules. Finally the composite

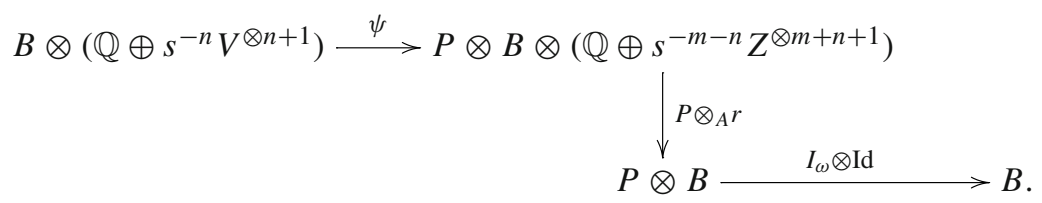

gives a morphism (of degree 0$)$ of $(B, d)$-module which is a retraction for the inclusion $B \rightarrow B \otimes\left(\mathbb{Q} \oplus s^{-n} V^{\otimes n+1}\right)$. This proves that msecat $\left(p^{\prime}\right) \leq n$.

Acknowledgements J.C. is supported by the Polish National Science Centre Grant 2016/21/ P/ST1/03460 within the European Unions Horizon 2020 research and innovation programme under the Marie SkłodowskaCurie Grant Agreement No. 665778 and by the Belgian Interuniversity Attraction Pole (IAP) within the framework "Dynamics, Geometry and Statistical Physics" (DYGEST P7/18). L.V. is partially supported by Portuguese Funds through FCT - Fundação para a Ciência e a Tecnologia, within the Project UID/MAT/00013/2013. 


\section{References}

1. Carrasquel-Vera, J.G.: The rational sectional category of certain maps. Ann. Sc. Norm. Super. Pisa Cl. Sci. XVII, 805-813 (2017)

2. Carrasquel-Vera, J.G.: The Ganea conjecture for rational approximations of sectional category. J. Pure Appl. Algebra 220(4), 1310-1315 (2016)

3. Carrasquel-Vera, J.G.: Rational methods applied to sectional category and topological complexity. Topological Complexity and Related Topics. Contemporary Mathematics American Mathematical Society. arXiv: 1703.02791 (to appear)

4. Farber, M.: Topological complexity of motion planning. Discret. Comput. Geom. 29(2), 211-221 (2003)

5. Félix, Y., Halperin, S., Lemaire, J.-M.: The rational LS category of products and of Poincaré duality complexes. Topology 37(4), 749-756 (1998)

6. Félix, Y., Halperin, S., Thomas, J.-C.: Rational homotopy theory, volume 205 of graduate texts in mathematics. Springer-Verlag, New York (2001)

7. Fernández Suárez, L., Ghienne, P., Kahl, T., Vandembroucq, L.: Joins of DGA modules and sectional category. Algebraic Geom. Topol. 6, 119-144 (2006)

8. González, J., Grant, M., Vandembroucq, L.: Hopf invariants for sectional category with applications to topological robotics, Preprint (2015) arXiv:1405.6891

9. Hess, K.: A proof of Ganea's conjecture for rational spaces. Topology 30(2), 205-214 (1991)

10. Lusternik, L., Schnirelmann, L.: Méthodes topologiques dans les problèmes variationnels, vol. 188. Hermann, Paris (1934)

11. Rudyak, Y.: On higher analogs of topological complexity. Topo.y Appl. 157(5), 916-920 (2010)

12. Schwarz, A.: The genus of a fiber space. AMS Transl 55, 49-140 (1966)

13. Sullivan, D.: Infinitesimal computations in topology. Inst. Hautes Études Sci. Publ. Math. 47, 269-331 (1977) 